\title{
C-reactive protein but not atrial dysfunction predicts recurrences of atrial fibrillation after cardioversion in patients with preserved left ventricular function Federico Lombardi ${ }^{a}$, Fabrizio Tundo ${ }^{a}$, Sebastiano Belletti ${ }^{a}$, Antonio Mantero ${ }^{a}$ and Gian Vico Melzi d'Eril
}

Objectives Maintenance of sinus rhythm after cardioversion of atrial fibrillation is a major clinical challenge also in patients with preserved left ventricular function. Subclinical inflammation and atrial strain have been recognized as important contributors to atrial fibrillation onset and perpetuation. Aim of the study was to compare the predictive role of $\mathrm{C}$-reactive protein (CRP) and indices of atrial dysfunction in relation to subacute arrhythmic recurrence rate in patients with persistent atrial fibrillation and normal left ventricular ejection fraction (LVEF).

Methods We studied 53 patients with a mean LVEF of $58.7 \pm 6 \%$. Left atrial diameter and area, left atrial auricle emptying velocity, N-terminal pro-b-type natriuretic peptide (NT-proBNP) and CRP levels were determined few hours before electrical cardioversion. NT-proBNP and CRP levels were also measured $1 \mathrm{~h}$ and 3 weeks after cardioversion.

Results Subacute atrial fibrillation recurrences were documented in $18(33.9 \%)$ patients. Whereas none of the parameters reflecting atrial dysfunction predicted arrhythmic outcome, higher CRP levels $(>3.0 \mathrm{mg} / \mathrm{l})$ were significantly associated with atrial fibrillation recurrences [odds ratio (OR): 1.6; 95\% confidence interval (Cl): 1.4-2.5; $P=0.031$. No changes in CRP levels were evident after cardioversion independently of underlying rhythm. On the contrary, NT-proBNP levels, which were correlated with left atrial auricle emptying velocity, significantly decreased only in patients who maintained sinus rhythm (from $638 \pm 329$ to $295 \pm 261 \mathrm{pg} / \mathrm{ml} ; P<0.001)$.

Conclusion The present study demonstrates that in patients with persistent atrial fibrillation and preserved LVEF, CRP level is an independent predictor of atrial fibrillation subacute recurrence rate, whereas none of the indices of atrial dysfunction is associated with arrhythmic outcome. NT-proBNP levels reflect, instead, the hemodynamic alterations secondary to arrhythmia presence. J Cardiovasc Med 9:581-588 (c) 2008 Italian Federation of Cardiology.

Journal of Cardiovascular Medicine 2008, 9:581-588

Keywords: atrial fibrillation, electrical cardioversion, natriuretic peptide, structural remodeling, subclinical inflammation

${ }^{a}$ Cardiology Laboratory, Department of Medicine, Surgery and Odontoiatry, Hospital San Paolo, University of Milan and ${ }^{\mathrm{b}}$ Clinical Biochemistry Laboratory, Department of Medicine, Surgery and Odontoiatry, Hospital San Paolo, University of Milan, Milan, Italy

Correspondence to Federico Lombardi, MD, FESC, MHRS, Cardiology Laboratory, Department of Medicine, Surgery and Odontoiatry, Hospital San Paolo, University of Milan, Via A. Di Rudini 8, 20142 Milan, Italy Tel: +39 0250323145; fax: +39 0250323145;

e-mail: Federico.Lombardi@unimi.it

\section{Introduction}

Maintenance of sinus rhythm after cardioversion of atrial fibrillation is a major clinical challenge. It is a common experience that more than one-third of patients undergoing cardioversion present early recurrences despite appropriate antiarrhythmic therapy [1-3]. Thereafter, the incidence of recurrences substantially and progressively decreases. Identification of factors favoring atrial fibrillation recurrence, through a better comprehension of arrhythmia pathophysiology [4,5], could suggest new strategies aimed to improve our success in maintaining sinus rhythm.

In the past, classical factors known to influence atrial fibrillation history were the presence of left ventricular dysfunction, left atrial enlargement, arrhythmia duration and history of hypertension [1]. More recently, other factors have been found to play a major role in atrial fibrillation onset and perpetuation. Among them, subclinical inflammation [6,7] and atrial strain [5] seem to be the most important ones. Indeed, several reports have indicated that inflammation is not the only central contributor to atherothrombosis but also there is a mechanistic link between inflammatory processes and development of atrial fibrillation [6]. Moreover, strain-mediated changes in atrial volume and function have been identified as critical components of atrial structural remodeling [5].

The aim of this study was to compare the predictive role of indices of atrial dysfunction including left atrial diameter (LAD), left atrial area (LAA), left atrial auricle emptying velocity (LAAEV), N-terminal pro-b-type 
Table 1 Demographic and clinical characteristics at baseline evaluation ( $\mathrm{T}-0)$ for all patients as a whole and in relation to AF recurrence during 3-week follow-up (T-3W)

\begin{tabular}{|c|c|c|c|c|}
\hline & All population $(n=53)$ & $\mathrm{AF}$ recurrences $(n=18)$ & SR maintenance $(n=35)$ & $P$ value \\
\hline Age (years) & $67 \pm 7$ & $65 \pm 6.6$ & $67.8 \pm 6.9$ & NS \\
\hline Sex $(M / F)$ & $37 / 16$ & $12 / 6$ & $25 / 10$ & NS \\
\hline LVEF (\%) & $58.7 \pm 5.8$ & $59 \pm 6.2$ & $58.5 \pm 5.6$ & NS \\
\hline LA enlargement (\%) & $39(71.7 \%)$ & $12(66.6 \%)$ & $27(77.1 \%)$ & NS \\
\hline $\mathrm{LA}$ area $\left(\mathrm{mm}^{2}\right)$ & $24 \pm 2.6$ & $23.6 \pm 3.2$ & $24.3 \pm 2.4$ & NS \\
\hline LAA emptying velocity $(\mathrm{cm} / \mathrm{s})$ & $30(20-30)$ & $30(20-30)$ & $30(20-30)$ & NS \\
\hline History of hypertension (\%) & $33(62.2 \%)$ & $10(55.5 \%)$ & $23(65.7 \%)$ & NS \\
\hline Diabetes (\%) & $3(5.6 \%)$ & $1(5.5 \%)$ & $2(5.7 \%)$ & NS \\
\hline History of $\mathrm{AF}(\%)$ & $23(43.4 \%)$ & $8(44.45)$ & $15(42.8 \%)$ & NS \\
\hline History of smoking (\%) & $15(28.3 \%)$ & $7(38.8 \%)$ & $8(22.8 \%)$ & NS \\
\hline Warfarin (\%) & $53(100 \%)$ & $18(100 \%)$ & $35(100 \%)$ & NS \\
\hline Beta-blockers (\%) & $17(32 \%)$ & $6(33.3 \%)$ & $11(31.4 \%)$ & NS \\
\hline ACEl/ARBs (\%) & $33(62.2 \%)$ & $11(61.1 \%)$ & $22(62.8 \%)$ & NS \\
\hline$A A-1 C(\%)$ & $3(5.6 \%)$ & $1(5.5 \%)$ & $2(5.7 \%)$ & NS \\
\hline Digoxin $(\%)$ & $4(7.5 \%)$ & $2(11.1 \%)$ & $2(5.7 \%)$ & NS \\
\hline Amiodarone (\%) & $29(54.7 \%)$ & $9(50 \%)$ & $20(57.1 \%)$ & NS \\
\hline $\mathrm{CRP}(\mathrm{mg} / \mathrm{l})$ & $2.4(0.91-3.70)$ & $3.70(2.2-5.9)$ & $1.96(0.6-3.0)$ & $P=0.014$ \\
\hline NT-proBNP values $(\mathrm{pg} / \mathrm{ml})$ & $664 \pm 465$ & $735 \pm 370$ & $638 \pm 329$ & NS \\
\hline
\end{tabular}

CRP data are presented as median interquartile range (IQR). AA-1C, class $1 \mathrm{C}$ antiarrhythmic drugs; ACEI/ARBs, angiotensin conversion enzyme inhibitors/angiotensin receptor blockers; AF, atrial fibrillation; LVEF, left ventricle ejection fraction; LA, left atrium; LAA, left atrium appendage; NT-proBNP, N-terminal pro-b-type natriuretic peptide; SR, sinus rhythm; T-0, baseline evaluation; T-3W, 3-week evaluation.

natriuretic peptide (NT-proBNP) levels and of subclinical inflammation (CRP) on subacute atrial fibrillation recurrences in hypertensive patients with persistent atrial fibrillation and preserved left ventricular ejection fraction (LVEF).

\section{Methods \\ Study population}

From our outpatient arrhythmia clinic, we prospectively enrolled 53 consecutive patients with persistent atrial fibrillation, preserved ventricular function (defined as a LVEF $>45 \%$ ), indication and consent for electrical cardioversion. A diagnosis of stage 1 hypertension or high normal blood pressure was present in, respectively, 62 and $21 \%$ of cases, lone atrial fibrillation in $14 \%$ of subjects. Patients with ischemic or valvular cardiomyopathy, NYHA functional classes II-IV, signs of acute or chronic inflammatory disease, malignancies, significant renal or hepatic failure, valvular cardiomyopathy, left ventricular hypertrophy and thyrotoxicosis were excluded from a possible inclusion in this study. The cut-off value of LVEF greater than $45 \%$ (measured during ongoing arrhythmia) was selected to include most of the patients without organic cardiomyopathy and heart failure symptoms. It is worth noting that of the six patients with ejection fraction of less than $55 \%$, only two had ejection fraction below $50 \%$.

All patients were on anticoagulant therapy with warfarin targeting an INR range $2-3$. The clinical characteristics and concomitant medical therapy are described in Table 1 .

The study protocol was approved by our Institutional Ethical Committee. Every patient signed a written informed consent.

\section{Study design}

Before electrical cardioversion (baseline evaluation time: T-0; Table 2), every patient underwent physical examination, transthoracic (TTE) and transesophageal (TEE) echocardiographic evaluation to determine LAD, LAA, LAAEV and LVEF. TTE was performed within 2 weeks before cardioversion, whereas TEE on the same morning of the procedure. Blood samples for NT-proBNP and high-sensitivity CRP measurements were collected immediately before and $1 \mathrm{~h}$ after cardioversion as well as at 3-week follow-up. NT-proBNP levels were determined by an electrochemiluminescence immunoassay on an Elecsys analyzer (Roche Diagnostics, Mannheim, Germany). Normal cut-off values were less than $264 \mathrm{pg} / \mathrm{ml}$ in men and less than $337 \mathrm{pg} / \mathrm{ml}$ in women. The analytical range extended from 5 to $35000 \mathrm{pg} / \mathrm{ml}$. Intraassay (interassay) $\%$ coefficient of variation was $3.5 \%$ $(4.1 \%)$ and $2.9 \%(3.7 \%)$ at concentrations of 251 and $805 \mathrm{pg} / \mathrm{ml}$, respectively. High-sensitivity CRP levels were determined by immunonephelometric assay using BN II System (Dade Behring, Marburg, Germany). Normal cut-off value was less than $3 \mathrm{mg} / \mathrm{l}$. The detection

\section{Table 2 Study protocol design}

\begin{tabular}{lccccc}
\hline & T-0 & T-1h & T-1W & T-2W & T-3W \\
$(n=53)$ & $(n=53)$ & $(n=53)$ & $(n=53)$ & $(n=53)$ \\
\hline $\begin{array}{l}\text { History collection } \\
\text { Physical examination }\end{array}$ & $\bullet$ & & & & \\
ECG & $\bullet$ & $\bullet$ & & & $\bullet$ \\
TTE & $\bullet$ & & & & $\bullet$ \\
TEE & $\bullet$ & & $\bullet$ & $\bullet$ & \\
Phone contact & & & $\bullet$ & & $\bullet$ \\
\hline
\end{tabular}

$\mathrm{CV}$, cardioversion; T-0, baseline evaluation before $\mathrm{CV} ; \mathrm{T}-1 \mathrm{~h}$, evaluation $1 \mathrm{~h}$ after CV; T-1W, T-2W, T-3W, evaluation, respectively, 1, 2 and 3 weeks after CV; TTE, transthoracic echocardiography; TEE, transesophageal echocardiography. ${ }^{\mathrm{a}}$ Blood sample collection at $\mathrm{T}-1 \mathrm{~h}$ was performed just for 19 subjects. 
limit was $0.175 \mathrm{mg} / \mathrm{l}$. Intraassay (interassay) coefficient of variation was $3.2 \%(3.8 \%)$ and $2.5 \%(3.5 \%)$ at concentrations of 0.5 and $26 \mathrm{mg} / \mathrm{l}$, respectively.

LVEF was calculated with Simpson method. The left atrial enlargement was defined as a diameter of greater than $40 \mathrm{~mm}$ measured in $\mathrm{M}$-mode in long-axis parasternal view or as LAA of greater than $20 \mathrm{~cm}^{2}$ measured in four-chamber apical view as described in the American Society of Echocardiography guidelines [8]. LAAEV was measured by Pulse Wave Doppler analysis during transesophageal examinations. Values less than $25 \mathrm{~cm} / \mathrm{s}$ were considered abnormal [9].

Electrical cardioversion was performed in fasting state during deep sedation with intravenous propofol (1-2 mg/kg). Bifasic DC shock (LifePak 12 defibrillator; Medtronic Inc., Minneapolis, USA) was delivered with rising energies when needed, starting from $100 \mathrm{~J}$ (single shock in almost all cases). In a subgroup of patients ( $n=19$; $35.8 \%$ ), a second blood sample was collected $1 \mathrm{~h}$ after cardioversion and sinus rhythm recovery to evaluate the possible effects of cardioversion on NT-proBNP levels (T-1h).

Patients' follow-up was planned with weekly phone contacts to investigate patient's symptoms in the first 2 weeks after cardioversion (time: T-1W; T-2W) and an ambulatory visit with ECG recording, 3 weeks after cardioversion (T-3W). Recurrences within this time frame were considered as subacute recurrences. During the medical control, blood samples were collected for the final CRP and NT-proBNP determination. All patients were also advised to contact our outpatient clinic when experiencing palpitations or shortness of breath during the 3-week follow-up period.

\section{Statistical analysis}

Data are presented as mean $\pm \mathrm{SD}$ or median and interquartile range (IQR) when asymmetrical distribution was observed. Normality was assessed with KolmogorovSmirnov test. Continuous variables were compared with unpaired or paired Student's $t$-test as needed (for normally distributed values) and Mann-Whitney or Wilcoxon rank sum test. Categorical variables were compared with $\chi^{2}$-test or Fisher's exact test. Correlation between variables was calculated with Pearson's and Spearmann's coefficients. Variables associated with atrial fibrillation recurrences were investigated with logistic regression analysis. OR is reported with 95\% CI. To achieve discrimination ability of CRP values to predict atrial fibrillation recurrences, a receiver operating characteristics (ROC) curve was plotted and the optimal cut-off value for CPR in minimizing the total number of false results and the area under the curve were calculated. A two-sided $P$ value of 0.05 was considered significant. All analyses were performed with software SPSS (Release 13.0, SPSS Inc., Chicago, USA).

\section{Results}

The mean age of patients was $67 \pm 7$ years, $69.8 \%$ were men. Left atrium enlargement enlargement was present in 39 (72\%) patients. Mean LVEF and LAA area were, respectively, $58.7 \pm 6 \%$ and $24 \pm 2.6 \mathrm{~cm}^{2}$. LAAEV was $30 \mathrm{~cm} / \mathrm{s}$ (median value; IQR: $20-30 \mathrm{~cm} / \mathrm{s}$ ). Arrhythmia duration ranged from 1 month to 1 year (median duration: 3 months). In $43 \%$ of the patients, a previous episode of atrial fibrillation was managed with electrical cardioversion.

In all patients sinus rhythm was successfully restored by electrical cardioversion. As expected, this maneuver was accompanied by a significant prolongation of mean RR interval (from $684 \pm 146$ to $946 \pm 138 \mathrm{~ms} ; P<0.01$ ). No adverse events were associated with the procedure. During a 3-week follow-up, 18 patients (33.9\%) showed atrial fibrillation recurrences. In most instances, no symptoms were reported. In two cases, in which symptoms before the programmed ambulatory visit were reported, an early atrial fibrillation recurrence was documented.

No significant differences were detectable between the two groups of patients in relation to mean age, sex, antiarrhythmic medication or other clinical data (Table 1).

\section{Atrial fibrillation recurrences in relation to atrial dysfunction}

At baseline evaluation (Table 1), none of the parameters reflecting atrial enlargement or dysfunction predicted atrial fibrillation recurrences. LAD, LAA, LAAEV measurements were similar in patients who maintained sinus rhythm and in patients with subacute arrhythmia recurrences.

NT-proBNP mean values $(664 \pm 465 \mathrm{pg} / \mathrm{ml})$ before cardioversion (T-0) were well above the reference normal values of our laboratory (see Methods). Of notice was the finding that abnormally elevated values were observed in all patients but two, in spite of preserved LVEF and absence of any echocardiographic evidence of left ventricular hypertrophy. When NT-proBNP levels were analyzed in relation to atrial fibrillation recurrences, no significant differences were observed in patients with subacute atrial fibrillation recurrences $(735 \pm 370 \mathrm{pg} / \mathrm{ml})$ in comparison with those who maintained sinus rhythm (638 \pm $329 \mathrm{pg} / \mathrm{ml}$ ). On the contrary, persistence of sinus rhythm was associated with a significant reduction of NT-proBNP (from $638 \pm 329$ to $295 \pm 261 \mathrm{pg} / \mathrm{ml} ; P<0.001$ ), whereas no decrease in peptide concentration was observed in patients with subacute arrhythmia recurrences (from $735 \pm 370$ to $757 \pm 421 \mathrm{pg} / \mathrm{ml} ; P=0.86$, see Fig. 1 ). We also observed that the cardioversion procedure and recovery of sinus rhythm determined a small but significant reduction (from $691 \pm 355$ to $618 \pm 311 \mathrm{pg} / \mathrm{ml} ; P=0.009$; Fig. 2) in NT-proBNP levels detectable within $1 \mathrm{~h}$ from the intervention (T-1h). 
Fig. 1

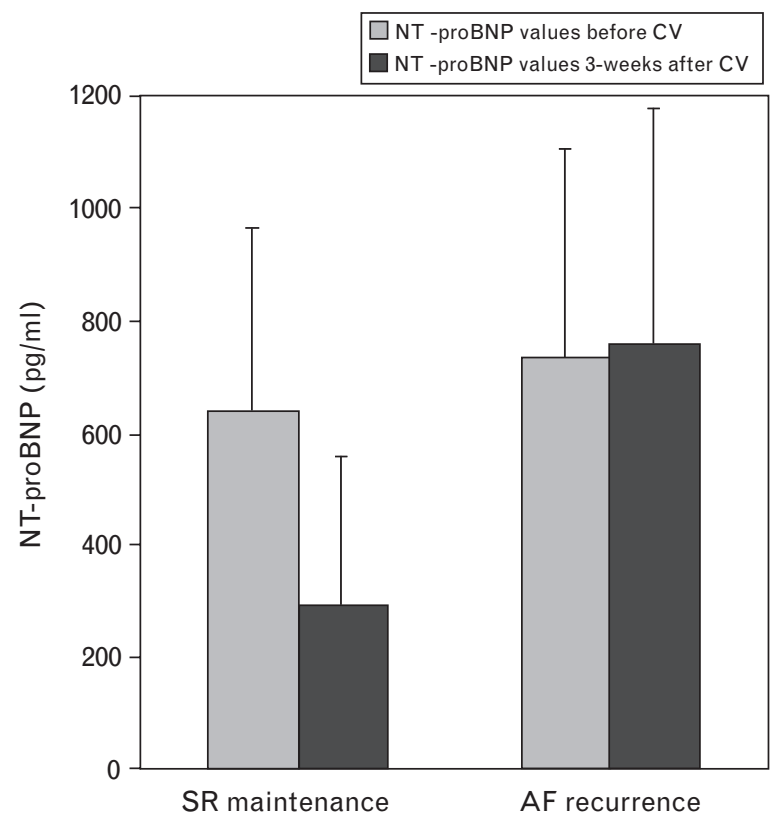

Rhythm at 3 weeks after CV

$\mathrm{N}$-terminal pro-b-type natriuretic peptide (NT-proBNP) values before and 3 weeks after electrical cardioversion (CV) in patients with sinus rhythm (SR) maintenance and atrial fibrillation (AF) recurrence. Data are presented as mean $\pm \mathrm{SD}$.

Fig. 2

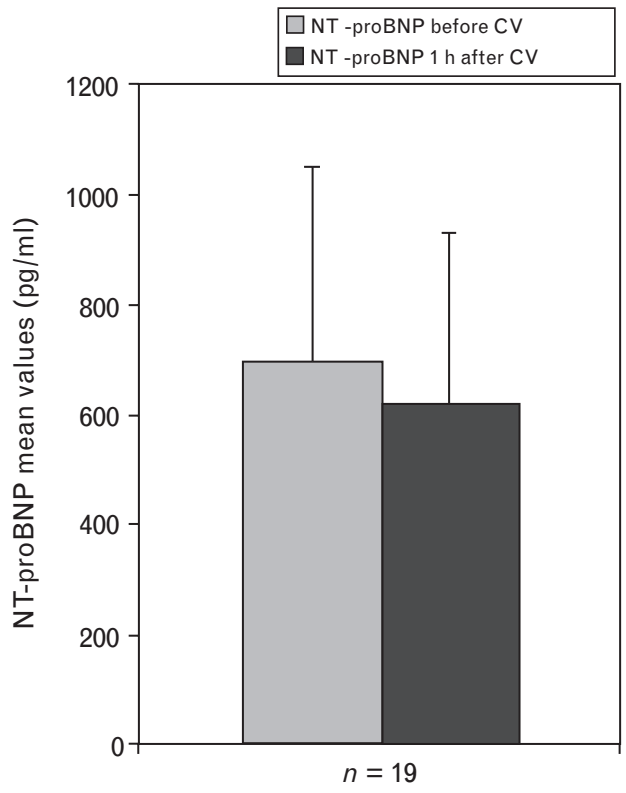

$\mathrm{N}$-terminal pro-b-type natriuretic peptide (NT-proBNP) values before and $1 \mathrm{~h}$ after successful electrical cardioversion and restoration of sinus rhythm (19 patients). Data are presented as mean \pm SD.
Fig. 3

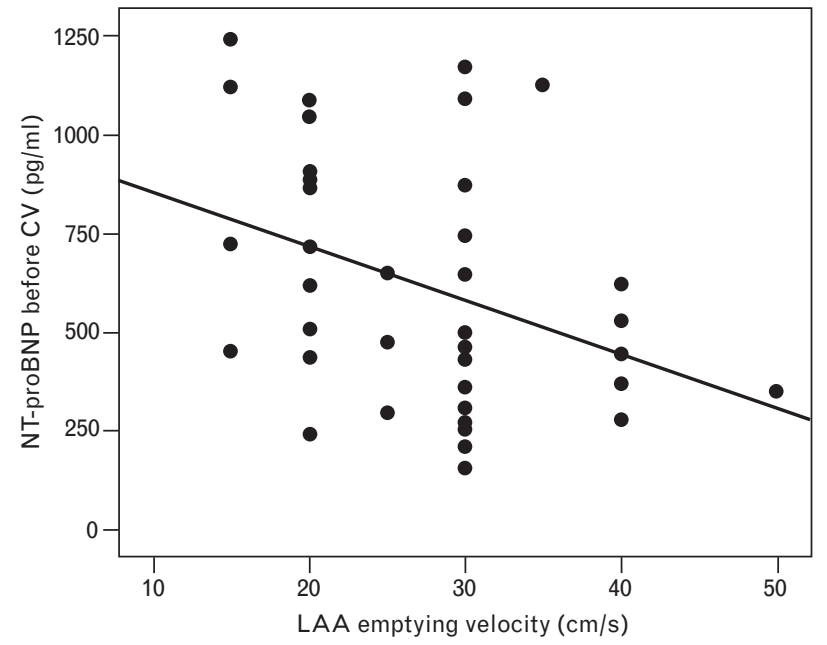

Correlation between $\mathrm{N}$-terminal pro-b-type natriuretic peptide (NT-proBNP) values before electrical cardioversion (CV) and left atrial appendage (LAA) emptying velocity assessed at transesophageal echocardiography (TEE) during atrial fibrillation $(r=0.54 ; P<0.001)$.

NT-proBNP levels were correlated with age $(r=0.3$, $P=0.04)$ and with LAAEV $(r=-0.54, P<0.001$, Fig. 3). This correlation was even more evident when correcting NT-proBNP values for age $(r=-0.58$, $P<0.001)$. No correlation was detectable between NT-proBNP levels and atrial fibrillation duration.

\section{C-reactive protein values and effects of cardioversion}

Median CRP level before cardioversion was 2.4 (IQR: 0.91-3.70). When CRP levels were analyzed in relation to arrhythmic outcome, higher values were observed in patients with subacute arrhythmia recurrences $(3.70 \mathrm{mg} / \mathrm{dl}$, IQR: $2.2-5.9)$ than in those who maintained sinus rhythm $(1.96 \mathrm{mg} / \mathrm{dl}$, IQR: $0.6-3.0 ; P=0.014$, Fig. 4). Of interest was the finding that patients in the highest quartile of CRP levels had an atrial fibrillation recurrence rate of $71 \%$, whereas in those in the lowest quartile the recurrence rate was $12 \%$.

At variance with NT-proBNP levels, no differences were observed in CRP levels measured before and after electrical cardioversion either in patients who maintained sinus rhythm (from $1.96 \mathrm{mg} / \mathrm{l}$, IQR: $0.6-3.0$ to $1.90 \mathrm{mg} / \mathrm{l}$, IQR: $1.0-2.9 ; P=0.31$ ) or with relapsing arrhythmia (from $3.7 \mathrm{mg} / \mathrm{l}$, IQR: $2.2-5.9$ to $3.35 \mathrm{mg} / \mathrm{l}$ IQR: $1.6-7.1 ; P=0.25$ ).

CRP levels did not show significant correlations with any parameter reflecting atrial dysfunction including LAD, LAA, LAARV and NT-proBNP levels.

\section{Predictive value of NT-proBNP and C-reactive protein levels}

In multinomial logistic regression analysis model performed to identify predictors of atrial fibrillation 
Fig. 4

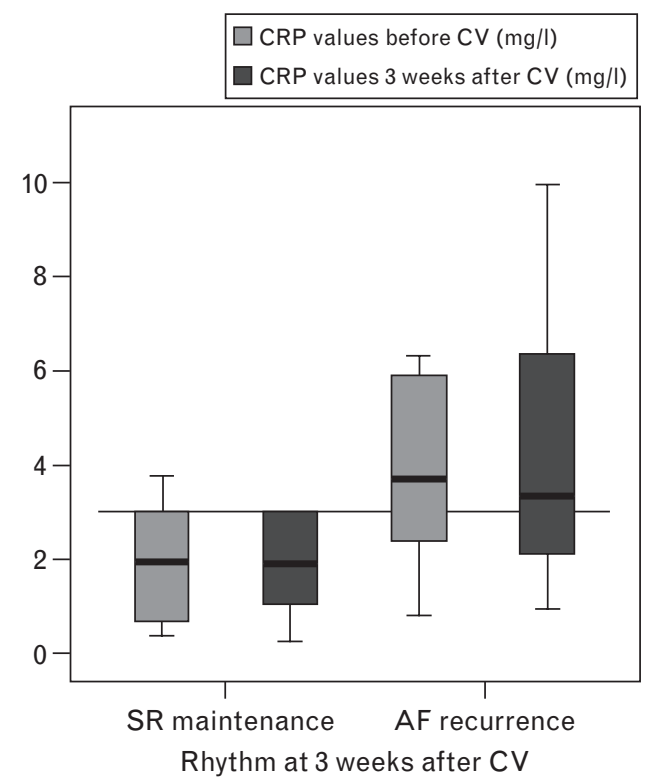

C-reactive protein (CRP) values before and 3 weeks after cardioversion (CV) in patients with sinus rhythm (SR) maintenance and atrial fibrillation (AF) recurrence. Horizontal line is set to cut-off value (3 $\mathrm{mg} / \mathrm{l})$. Data are presented as median (interquartile range).

recurrence, variables classically associated with arrhythmia recurrence like age, LAD, LAA, LAAEV, NTproBNP level, history of previous atrial fibrillation, arrhythmia duration or antiarrhythmic medication failed to reach a statistical significance. On the contrary, CRP levels were significantly associated with arrhythmia recurrence with an OR of 1.6 (95\% CI: 1.04-2.5; $P=0.031$ ). When considering the variable as dichotomic, splitting GRP serum concentration obtained at T-0 analysis by the cut-off values of $3 \mathrm{mg} / \mathrm{l}$, a significant difference in atrial fibrillation recurrence rate between the two groups $(P=0.027)$ was observed. A CRP value of at least $3.0 \mathrm{mg} / \mathrm{l}$ presented a positive and negative predictive value of, respectively, 58 and $78 \%$ for early atrial fibrillation recurrence. The percentage of atrial fibrillation recurrence was, respectively, 12, 25, 33 and $71 \%$ in the four quartiles of CRP distribution, ranging from lower to higher values $(P<0.001$; Fig. 5$)$. By constructing a ROC curve for CRP and arrhythmia recurrence, we identified an optimal cut-off value of $3.6 \mathrm{mg} / \mathrm{dl}$ (similar to upper quartile distribution value of $3.7 \mathrm{mg} / \mathrm{l}$ ) minimizing false positive and negative results (ROC area: 0.76 ), obtaining positive and negative predictive values of, respectively, 64 and $81 \%$ (Fig. 6).

\section{Discussion}

The main results of this study were the following: in patients with persistent atrial fibrillation and preserved LVEF, none of the atrial parameters reflecting atrial dysfunction or strain was associated with atrial fibrillation
Fig. 5

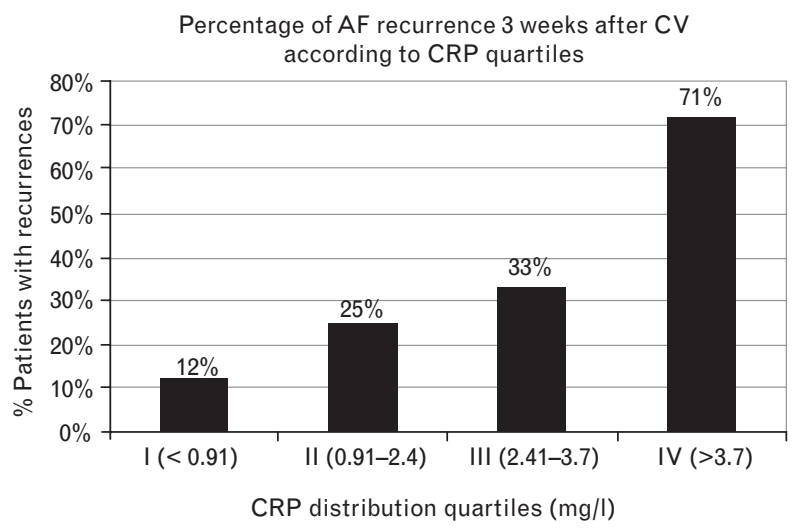

Percentage of atrial fibrillation (AF) recurrence according to $\mathrm{C}$-reactive protein $(\mathrm{CRP})$ distribution quartiles $(P<0.001)$.

recurrences; CRP levels measured before cardioversion predicted arrhythmia recurrences and were not modified by the underlying rhythm; NT-proBNP levels were inversely correlated with LAAEV and significantly decreased after recovery of sinus rhythm.

\section{Atrial strain and atrial fibrillation}

In the present study, LAD, LAA, LAAEV and NTproBNP levels were used as indices of atrial strain associated with the abnormal hemodynamic function that

Fig. 6

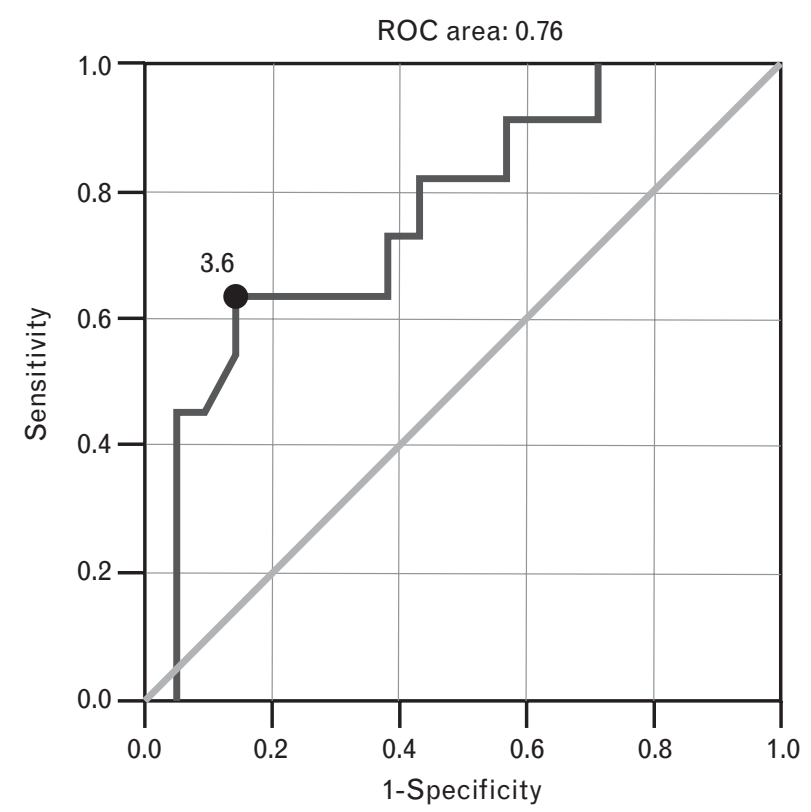

Receiver operating characteristic (ROC) for C-reactive protein (CRP) and arrhythmia recurrence. An optimal cut-off value of $3.6 \mathrm{mg} / \mathrm{dl}$ (similar to upper quartile distribution value of $3.7 \mathrm{mg} / \mathrm{l})$ minimizing false positive and negative results was selected, obtaining positive and negative predictive values of, respectively, 64 and $81 \%$. 
characterize atrial fibrillation patients [10]. In spite of a normal or LVEF and in absence of any echocardiographic evidence of left ventricular hypertrophy, a left atrium enlargement was present in more than $70 \%$ of the patients and a reduced LAAEV was measured in all subjects. NT-proBNP levels were elevated in all but two patients. It is well known that B-type natriuretic peptide is released in response to myocite stretch by ventricular myocites [10] and is considered the best accepted diagnostic tool in the evaluation of dyspnea in the emergency department [11]. In asymptomatic middle-aged persons, the level of B-type natriuretic peptide is an independent predictor of death, heart failure, atrial fibrillation and stroke in a 5 -year follow-up [12].

In our study, carried out in a selected group of patients with preserved LVEF and a history of hypertension in more than $50 \%$ of cases, high levels of NT-proBNP were likely to reflect factors related to atrial fibrillation rather than a subclinical heart disease. It is possible that diastolic dysfunction due to rhythm irregularity and loss of atrial contribution to ventricular filling as well as the increased atrial strain associated with atrial fibrillation might have caused NT-proBNP level elevation. The significant correlation between LAAEV and NT-proBNP is consistent with this hypothesis and confirms the sensitivity of this parameter to reflect the functional severity of hemodynamic alterations associated with atrial fibrillation. This interpretation is also supported by the fact that after cardioversion, NT-proBNP levels significantly decreased only in patients who maintained sinus rhythm.

The reduction in NT-proBNP levels after successful cardioversion observed in the present study is in agreement with previous observation $[13,14]$ that reported a reduction in NT-proBNP levels after recovery of sinus rhythm in patients with preserved LVEF and paroxysmal or persistent atrial fibrillation. In both studies, NTproBNP levels were not related to arrhythmia duration and were not correlated with atrial dimension. In our study, in which we measured, in addition, LAAEV as an indirect index of atrial mechanical dysfunction, a significant inverse correlation between NT-proBNP levels and LAAEV was instead observed. The consistent finding that NT-proBNP levels were not increased by DC shock but significantly decreased immediately after restoration of sinus rhythm opens, in our opinion, new possibility for the utilization of this parameter as a noninvasive marker of arrhythmia burden in patients with either asymptomatic or short-lasting episodes of atrial fibrillation. Detection of asymptomatic recurrences is a major clinical problem: one could hypothesize that in patients with a history of atrial fibrillation, the demonstration of normal NT-proBNP levels could confirm the presence of an arrhythmia free period.

\section{Subclinical inflammation and atrial fibrillation}

The association between atrial fibrillation and inflammation is supported by the frequent association between inflammatory disease such as myocarditis or pericarditis and atrial fibrillation [6,7]. The increased frequency of atrial fibrillation after cardiac surgery [15] is commonly interpreted as an indirect evidence for this pathophysiological hypothesis [6,7]. Presence of inflammatory infiltrates and oxidative damage has been demonstrated in atrial biopsies of atrial fibrillation patients, thus providing a histopathological evidence [16].

In the last decade, several studies [6,7,17], by using markers of inflammation such as CRP and interleukin-6, have indicated higher levels of both the parameters in patients with atrial fibrillation than in controls in sinus rhythm. Moreover, patients with persistent atrial fibrillation have been found to present higher CRP levels than the patients with paroxysmal atrial fibrillation in relation to a longer arrhythmia duration and atrial dimension, thus suggesting a link between inflammation and structural remodeling [5,6]. Regarding this latter factor, it is important to recognize that angiotensin II has been identified as a potential contributor to inflammation also in atrial fibrillation patients $[6,7,18]$. Thus, inflammation may represent one of the pathways by which the activation of the renin-angiotensin-aldosterone system concurs to the development and perpetuation of atrial fibrillation, linking neuro-humoral alterations, structural remodeling and susceptibility to atrial fibrillation [5-7].

In the present study, carried out in patients with preserved LVEF, history of hypertension and ACE inhibitor treatment in about two out of three cases, we observed higher CRP levels in patients with atrial fibrillation recurrence, thus confirming the predictive role of this marker of subclinical inflammation also in patients with persistent atrial fibrillation [19-22]. Moreover, we observed that neither restoration of a more physiological hemodynamic condition nor persistence of atrial fibrillation determined any significant change in CRP levels during the 3-week period of observation.

These findings are in agreement with previous reports $[19,21,22]$ that have indicated CRP as a predictor for recurrence of atrial fibrillation after successful cardioversion. In the above as well as in our study, CRP levels were not correlated with atrial dimension, arrhythmia duration or antiarrhythmic drug administration. Watanabe et al. [20] reported, in addition, that CRP levels were also predictors of successful cardioversion and found a significant correlation between CRP levels and extent of coronary artery disease, smoking, diabetes and atrial fibrillation duration. In the same study, the authors observed a reduction in CRP levels after cardioversion only in patients with atrial fibrillation recurrences, whereas CRP levels remained unchanged in those 
subjects who maintained sinus rhythm during the entire follow-up period. It is our opinion that major differences in the study population might explain these different results: the lower success rate of cardioversion and the higher atrial fibrillation recurrence rate reported in Watanabe study support, in our opinion, this interpretation.

In conclusion, all these findings are consistent with the concept that CRP levels might reflect the structural changes associated with atrial fibrillation and that subclinical inflammation is one of its most important components. This interpretation is confirmed by the study of Ellinor et al. [23], who analyzed CRP and interleukin-6 levels in lone and hypertensive atrial fibrillation patients. These authors found higher values in the latter group but at variance with our study did not observe any significant correlation between CRP levels and atrial fibrillation recurrences. Recently [24], higher CRP levels have been reported in patients with the first symptomatic episode of paroxysmal atrial fibrillation and found associated with atrial fibrillation recurrences as in our report in patients with persistent atrial fibrillation.

The presence of high CRP levels in patients with atrial fibrillation recurrences not only emphasizes the proarrhythmic role of subclinical inflammation but also opens new perspectives to consider CRP levels, which have also been associated with stroke incidence [25], as a therapeutical target. Preliminary results indicate that pharmacological treatments such as those with ACE inhibitors [26,27] and statins $[6,28]$ may exert a beneficial effect in atrial fibrillation patients by modulating those factors that are known to affect structural remodeling and have the inflammatory process as one of their major components. In this context, the potential beneficial effect of steroids and fish oil could also be considered. Dernellis and Panaretou [29] reported that methylprednisolone, an adjunct to amiodarone, significantly reduced arrhythmia recurrence rate in patients with persistent atrial fibrillation, whereas Calò et al. [30] showed that pretreatment with omega $n$-3 fatty acids reduced the incidence of postcardiac surgery atrial fibrillation in comparison with patients treated conventionally.

All the above data seem, therefore, to support the concept that pharmacological modulation of inflammatory mechanisms might be a potential beneficial strategy in atrial fibrillation patients.

\section{Study limitations}

The small number of patients enrolled in the study is, probably, its major limitation. The study, however, was restricted to a homogeneous group of subjects without evidence of organic cardiomyopathy, thus limiting the confounding effects of factors such as left ventricular dysfunction known to affect atrial parameters or NT-
proBNP levels. A second limitation is the fact that the study duration was limited to a 3-week period, which, however, accounts for the greatest rate of recurrences after cardioversion $[4,5]$. Subacute arrhythmia recurrence rate was indeed consistent with previous reports $[4,5]$ and an adequate logistic regression analysis model was used to evaluate the predictive value of CRP. Finally, it is important to recall that in this study, relapses identification was based on symptoms in the first 2 weeks and on ECG recordings at 3 weeks after cardioversion. This protocol timing could have underestimated the real incidence of atrial fibrillation recurrences by excluding asymptomatic episodes of short duration, whose effects on NT-proBNP and CRP values have not yet been determined.

\section{Conclusions}

The present study indicates that in patients with preserved LVEF and a history of hypertension, CRP levels rather than indices of atrial dysfunction predict arrhythmia recurrences. On the contrary, NT-proBNP levels, which significantly decrease after sinus rhythm recovery, appear to better reflect the hemodynamic alterations secondary to arrhythmia presence. The possibility of using NT-proBNP as a marker of atrial fibrillation burden in atrial fibrillation patients is a hypothesis that deserves further investigation.

\section{Acknowledgement}

The study was partially supported by 2004 and 2006 PRIN grants of MIUR.

There are no conflicts of interest.

\section{References}

1 Kannel WB, Abbott RD, Savage DD, McNamara PM. Epidemiologic features of atrial fibrillation: the Framingham study. N Engl J Med 1982; 306:1018-1022.

2 Tieleman RG, Van Gelder IC, Crijns HJ, De Kam PJ, Van Den Berg MP, Haaksma J, et al. Early recurrence of atrial fibrillation after electrical cardioversion: a result of fibrillation induced electrical remodeling of the atria? J Am Coll Cardiol 1998; 31:167-173.

3 Lafuente-Lafuente $\mathrm{C}$, Mouly S, Longàs-Tejero MA, Mahé I, Bergmann JF. Antiarrhythmic drugs for maintaining sinus rhythm after cardioversion of atrial fibrillation. Arch Intern Med 2006; 166:719-728.

4 Allessie MA, Konings K, Kirchhof C, Wijffels M. Electrophysiologic mechanisms of perpetuation of atrial fibrillation. Am J Cardiol 1996; 77:10A-23A.

5 Allessie MA, Boyden PA, Camm JA, Kleber AG, Lab MJ, Legato MJ, et al. Pathophysiology and prevention of atrial fibrillation. Circulation 2001; 103:769-777.

6 Engelmann MD, Svendsen $\mathrm{JH}$. Inflammation in the genesis and perpetuation of atrial fibrillation. Eur Heart J 2005; 26:2083-2092.

7 Boos JB, Anderson RA, Lip GYH. Is atrial fibrillation an inflammatory disorder? Eur Heart J 2006; 27:137-149.

8 Lang RM, Bierig M, Devereux RB, Flachskampf FA, Foster E, Pellikka PA, et al. Recommendations for chamber quantification: a report from the American Society of Echocardiography's Guidelines and Standards Committee and the Chamber Quantification Writing Group, developed in conjunction with the European Association of Echocardiography, a branch of the European Society of Cardiology. J Am Soc Echocardiogr 2005; 18:1440-1463.

9 Antonielli E, Pizzuti A, Palinkas A, Tanga M, Gruber N, Michelassi C, et al. Clinical value of left atrial appendage flow for prediction of long-term sinus rhythm maintenance in patients with nonvalvular atrial fibrillation. J Am Coll Cardiol 2002; 39:1443-1449. 
10 Mark DB, Felker GM. B-type natriuretic peptide: a biomarker for all seasons? N Engl J Med 2004; 350:718-720.

11 de Lemos JA, McGuire DK, Drazner MH. B-type natriuretic peptide in cardiovascular disease. Lancet 2003; 362:316-322.

12 Wang TJ, Larson MG, Levy D, Benjamin EJ, Leip EP, Omland T, et al. Plasma natriuretic peptide levels and the risk of cardiovascular events and death. N Engl J Med 2004; 350:655-663.

13 Wozawkoska-Kaplon B. Effect of sinus rhythm restoration on plasma brain natriuretic peptide in patients with atrial fibrillation. Am J Cardiol 2004; 93:1555-1558.

14 Buob A, Jung J, Siaplaouras S, Neuberger H-R, Mewis C. Discordant regulation of CRP and NT-proBNP plasma levels after electrical cardioversion of persistent atrial fibrillation. PACE 2006; 29:559-563.

15 Mathew JP, Fontes ML, Tudor JC, Ramsey J, Duke P, Mazer CD, et al. A multicenter risk index for atrial fibrillation after cardiac surgery. J Am Med Assoc 2004; 291:1720-1729.

16 Frustaci A, Chimenti C, Bellocci F, Morgante E, Russo MA, Maseri A. Histological substrates of atrial biopsies in patients with lone atrial fibrillation. Circulation 1997; 96:1180-1184.

17 Psychari SN, Apostolou TS, Sinos L, Hamodraka E, Liakos G, Kremastinos DT. Relation of elevated C-reactive protein and interleukin- 6 levels to left atrial size and duration of episodes in patients with atrial fibrillation. $\mathrm{Am} J$ Cardiol 2005; 95:764-767.

18 Tamarat R, Silvestre JS, Durie M, Levy BI. Angiotensin II angiogenic effect in vivo involves vascular endothelial growth factor and inflammation-related pathways. Lab Invest 2002; 82:747-756.

19 Malouf JF, Kanagola R, AIAtawi FO, Rosales AG, Davison DE, Murali NS, et al. High sensitivity $\mathrm{C}$-reactive protein: a novel predictor for recurrence of atrial fibrillation after successful cardioversion. J Am Coll Cardiol 2005; 46:1284-1287.

20 Watanabe E, Arakawa T, Uchiyama T, Kodama I, Hishida H. High-sensitivity $\mathrm{C}$-reactive protein is predictive of successful cardioversion for atrial fibrillation and maintenance of sinus rhythm after conversion. Int J Cardiol 2006; 108:346-353.

21 Wazni O, Martin DO, Marrouche NF, Shaaraoui M, Chung MK, Almahameed $\mathrm{S}$, et al. $\mathrm{C}$ reactive protein concentration and recurrence of atrial fibrillation after electrical cardioversion. Heart 2005; 91:1303-1305.

22 Zarauza J, Rodriguez Lera MJ, Farinas Alvarez C, Hernando JP, Ceballos B, Gutierrez B, et al. Relationship between C-reactive protein level and early recurrence of atrial fibrillation after electrical cardioversion. Rev Esp Cardiol 2006; 59:125-129.

23 Ellinor PT, Low A, Patton KK, Shea MA, MacRae CA. C-reactive protein in lone atrial fibrillation. Am J Cardiol 2006; 97:1346-1350.

24 Hatzinikolaou-Kotsakou E, Tziakas D, Hotidid A, Stakos D, Floros D, Papanas N, et al. Relation of C-reactive protein to the first onset and the recurrence rate in lone atrial fibrillation. Am J Cardio/ 2006; 97:659-661.

25 Thambidorai SK, Parakh K, Martin DO, Shah TK, Wazni O, Jasper SE, et al. Relation of $\mathrm{C}$-reactive protein correlates with risk of thromboembolism in patients with atrial fibrillation. Am J Cardiol 2004; 94:805-807.

26 Healey JS, Baranchuk A, Crystal E, Morillo CA, Garfinkle M, Yusuf S, et al. Prevention of atrial fibrillation with angiotensin-converting enzyme inhibitors and angiotensin receptor blockers: a meta-analysis. J Am Coll Cardiol 2005; 45:1832-1839.

27 Boos CJ, Lip GYH. Prevention of atrial fibrillation by angiotensinconverting-enzyme inhibitors and angiotensin II receptor blockers: some pathophysiological insights. J Am Coll Cardiol 2006; 47:889-890.

28 Ozaydin M, Varol E, Aslam SM, Kucuktepe Z, Dogan A, Ozturk M, Altinbas A. Effect of atorvastatin on the recurrence rates of atrial fibrillation after electrical cardioversion. Am J Cardiol 2006; 97:1490-1493.

29 Dernellis J, Panaretou M. Relationship between C-reactive protein concentrations during glucocorticoid therapy and recurrent atrial fibrillation. Eur Heart J 2004; 25:1100-1107.

30 Calò L, Bianconi L, Colivicchi F, Lamberti F, Loricchio ML, de Ruvo E, et al $\mathrm{N}-3$ fatty acids for the prevention of atrial fibrillation after coronary artery bypass surgery: a randomized controlled trial. J Am Coll Cardiol 2005; 45:1723-1728. 